(b) Under the condition (1.6), the expression $\sigma_{m, l}^{a, \beta}(x, y ; f)$ tends almost everywhere to $f(x, y)$, where $\sigma_{m, n}^{c, \beta}$ denotes the $(C, \alpha, \beta)$ means of the Fourier series of the function $f$, and the numbers $\alpha, \beta$ are positive. The proof follows the same line as that of Theorem 1 . We must only observe that the $(C, \alpha)$ kernel $K_{m}^{u}(u)$ satisfies for $0<\alpha<1$ the inequalities:

$\left|K_{m}^{u}(u)\right| \leqslant A(\alpha) m, \quad\left|K_{m}^{u}(u)\right| \leqslant A(\alpha) / m^{\alpha} u u^{u+1} \quad(m=1,2, \ldots, ; 0<u \leqslant \pi)$

analogous to (3.2). $(\boldsymbol{A}(\alpha)$ depends on $\alpha$ only).

Similarly we prove that, if $P(r, u)$ denotes Poisson's kernel, then

$$
\frac{1}{\pi^{2}} \int_{-i t}^{\pi} \int_{-\pi}^{\pi} f(x+u, y+v) P(r, u) P(\varrho, v) d u d v \rightarrow f(x, y)
$$

at almost every point $(x, y)$, provided that

$$
r \rightarrow 1, \quad \varrho \rightarrow 1, \quad(1-\varrho) /(1-r)<\lambda, \quad(1-r) /(1-\varrho)<\lambda .
$$

More generally, we have the following

Theorem 3. At almost every point $(x, y)$,

$$
\frac{1}{\pi^{2}} \int_{-\pi}^{\pi} \int_{-\pi}^{\pi} f(\xi+u, \eta+v) P(r, u) P(\varrho, v) d u d v \rightarrow f(x, y),
$$

provided that the conditions (4.2) are satisfied, and that the points with polar coordinates $(r, \xi),(\varrho, \eta)$ tend respectively to the points $(1, x),(1, y)$ along non tangential paths.

Similarly we may generalize Theorem $2^{9}$ ).

(c) Theorems 1 and 2 are true for the Fourier series of functions of $n$ variables. The proofs undergo no essential changes.

9) If the function $|f| \log ^{+}|f|$ is integrable over $Q$, we have (3.11), for $m$ and $n$ tending to $+\infty$ independently of each other. The function

satisfies the inequality

$$
\sigma^{*}(x, y ; f)=\underset{m, n}{\operatorname{Max}}\left|\sigma_{m, n}(x, y ; f)\right|
$$

$$
\left\{\iint_{Q}\left(o^{*}(x, y ; f)\right)^{1-\varepsilon} d x d y\right\}^{1 /(1-\varepsilon)}-\frac{A}{\varepsilon} \int_{Q} \int|f| \log +|f| d x d y+\frac{4}{\varepsilon}
$$

These results are impliritly contained in the paper quoted in footnote ${ }^{2}$ ).

\section{On the isomorphism and the equivalence of classes and sequences of sets ${ }^{1}$ ).}

By

\author{
Edward Szpilrajn (Warszawa).
}

Introduction. The notions which are the subject of this paper belong to the General Theory of Sets and particularly to its part which deals with classes and sequences of sets. It seems that one of the chief problems in this field is the investigation into such relations between classes (or sequences) of sets which are analogous to as important a relation between sets as the equality of powers.

The relationships in the domain of classes of sets are obviously more complicated than in the domain of sets; and so it is possible to define in a natural way many relations between classes of sets which are reflexive, symmetrical and transitive and which may be considered as analogous to the equality of powers. In this paper we examine four notions of this type: weak isomorphism, isomorphism ("iscmorphie algébro-logique" in terms of KuratowskiPosament $\left.{ }^{2}\right)$ ), total isomorphism, and equivalence ("double similarity" in terms of Whitehead-Russel! ${ }^{3}$ )).

Let us denote by $K$ and $L$ two classes of sets. If $K$ and $L$, considered as sets partially ordered by the relation of proper inclusion, are similar, then they are called weakly isomorphic. Further, we say that $K$ and $L$ are isomorphic when they have the same properties from the point of view of all finite operations upon sets (addition of two sets and complementation). Analogically, $K$ and $L$

1) Presented to the Polish Mathematical Society, Warsaw section, on May 6, 1938. Cf. the preliminary report Szpilrajn [6].

${ }^{2}$ Kuratowski-Posament [1], p. 282.

s) Whitehead-Russell [1], p. 84. Cf. also Sierpiński [1], p. 80, Stone [1], p. 91, szpilrajn [1], p. 306, [2] and [3]. 
are totally isomorphic when they have the same properties from the point of view of all operations upon sets (addition of an arbitrary finite or transfinite number of sets and complementation). Finally they are equivalent if, from the point of view of the General Theory of Sets, all their properties are identical ${ }^{1}$ ).

In $\S \S 1$ and 2 I state some general theorems on these relations and I give a fow of their applications to the study of point sets. In $\S 3$ I deal especially with enumerable sequences of setis. Before all I express the condition that two sequences of sets are isomorphic [or totally isomorphic] with the help of the characteristic function of a sequence of sets (3.2). Applying this condition and the properties of the characteristic function, I consider some questions concerning point sets, chiefly the problem of finding for a given sequence of sets an isomorphic [or totally isomorphic, or equivalent] sequence of sets of a special type e.g. Borel sets, projective sets, etc. $\left.{ }^{2}\right)$.

My paper on the characteristic function: szpilrajn [4] will be refered to as $\mathrm{CF}$.

Terminology and notation. By the term space we understand a wholly arbitrary abstract set. The letters $X, X$ and $Z$, when no additional explications are given, will always denote arbitrary spaces. If a metrical space $X$ is a Borel subset of a complete space, then $X$ is said to be Borel space. The interval $0 \leqslant t \leqslant 1$ is denoted by $I$; the space of irrational numbers by $N$, and the space which is the sum of $N I$ and of the set of all integers by $N^{*}$.

The Cantor set, i.e. the set of all numbers:

$$
t=2 \cdot\left(0, i_{1}, i_{2}, i_{3}, \ldots\right)_{3} \text {, where } i_{n}=0 \text { or } i_{n}=1 \text {, }
$$

is denoted by $O$. For $i=0,1$ and $n=1,2,3, \ldots$ we denote by $C_{n}^{i}$ the set of all num. bers $(*)$ such that $i_{n}=i$. Further, for each finite sequence $j_{1}, j_{2}, \ldots, j_{n}$ consisting of the numbers 0 and 1 , we denote by $\sigma_{j_{1}} j_{2} \ldots j_{n}$ the set of all numbers $\left(^{*}\right)$ such that $i_{k}=j_{k}$ for $k=1,2, \ldots n$, and we call this set interval of $C$. A subset of $O$ is openand-olosed in $C$ if and only if it is the sum of a finite number of intervals of $O$.

For each set $E \subset X$ we put $E^{0}=X-E$ and $E^{1}=E$. Consequently, we have $X-E^{i}=E^{1-i}$ for $i=0,1$.

For each sequence $e=\left\{E_{n}\right\}$ of subsets of a space $X$, we denote by $c_{e}(x)$ the characteristio function of $e$, i. e. the function which associates with each $x \in X$ a number $\left({ }^{*}\right.$ ), where $i_{n}=0$ or $i_{n}=1$ according as $x \in X-E_{n}$ or $x \in E_{n}$ (se日 CF 2.2).

1) Precise definitions will be given under 1.1. Let us remark that it is possible to define, for each oardinal number $n$, "the "1-isomorphism" analogous to "the n-additivity" in the sense of Tarski. See Tarski [2], p. 1.52.

2) A series of analogous problems concerning especially the equivalence was raised by Ulam and treated in the paper: Szpilrajn [2].
We call system of sets each class $E=\left\{E_{\xi}\right\}_{\xi \in \Xi}$ in which with each element ("index") $\xi$ belonging to a given set $\Xi$ there is associated a set $E_{\xi}$. (Similarly we may speak of a system $\left\{i_{\xi}\right\}_{\xi \in E}$ of numbers). In particular, when $E$ is the set of positive integers, one obtains a sequence of sets; more generally, if $\Xi$ consists of all ordinal numbers $\xi<\alpha$ (where $\alpha$ is a given ordinal number), we obtain a transfinite sequence.

$F$ being a biunivocal function, $F^{-1}$ denotes the inverse function. $\varrho$ being a relation, the set $A$ of all elements $a$ for which there exists an element $b$ such that $a \varrho b$ [such that $b \varrho a$ ] is called domain [counterdomain] of $\varrho$.

A class $K$ of subsets of $X$ is called $1^{0}$ complementative, $2^{\circ}$ additive, $3^{\circ}$ totally additive, $4^{0}$ multiplicative, $5^{0}$ totally multiplicative, when for every transfinite sequence $K$ of sets belonging to $K$ we have $1^{0} X-K_{1} \in \mathbf{K}, 2^{0} K_{1}+K_{2} \in \mathbf{K}$, $3^{0} K_{1}+K_{2}+\ldots+K_{\varepsilon}+\ldots \in \mathrm{K}, \quad 4^{0} \quad K_{1} \cdot K_{2} \in \mathrm{K}, \quad 5^{0} K_{1} \cdot K_{2} \cdot \ldots \cdot K_{\xi} \cdot \ldots \in \mathrm{K}$. Each complementative and additive [totally additive] class of sets is called a ring [a total ring]. The smallest ring [total ring] including a given class $\mathbf{K}$ of sets is denoted by $\mathbf{K}_{0}$ [by $K_{t}$ ]. Clearly, each ring [total ring] is multiplicative [totally multiplicative].

A function which associates with each set $E$ belonging to a complementative class $K$ (of subsets of $X$ ) a set $F(E)$ (contained in $Y$ ) is said to be com. plementative if we have $F(X-K)=Y-F(K)$ for $K \in \mathrm{K}$. Similarly we define the additivity, the total additivity, the multiplicativity and the total multiplicativity of $F$. Obviously each complementative and additive [totally additive] function of a set is multiplicative [totally multiplicative].

For each complementative function $F^{\prime}$ we always have: $F\left(E^{i}\right)=[F(E)]^{i}$ (for $i=0,1$ ).

\section{\$1. Isomorphisms and equivalence.}

1.1. Definitions and fundamental properties. A biunivocal transformation $F^{\prime}(K)$ of a class $\mathbf{K}$ of sets into a class $\mathbf{L}$ of sets is called weate isomorphism between $\mathrm{K}$ and $\mathrm{L}$ if given $K_{1}, K_{\mathbf{2}} \in \mathrm{K}$, we have $K_{1} \subset K_{2}$ if and only if $F\left(K_{1}\right) \subset F\left(K_{2}\right)$.

The condition of biunivocity may be omitted:

(i) A relation $\varrho$ is a weak isomorphism between $\mathbf{K}$ and $\mathbf{L}$ if and only if $1^{0}$ the domain and the counterdomain of $\varrho$ are $\mathbf{K}$ and $\mathbf{L}$ respectively, $2^{0}$ given $K_{1} \varrho L_{1}$ and $K_{2} \varrho L_{2}$, we have $K_{1} \subset K_{2}$ if and only if $L_{1} C L_{2}$.

In fact, it is easy to see that the condition $2^{0}$ implies the biunivocity of $\varrho$.

(ii) If $F(K)$ is a weak isomorphism between two rings [total rings $] \mathrm{K}$ and $\mathrm{L}$ of subsets of $X$ and $Y$, then: $1^{0} K=0$ if and only if $F(K)=0,2^{\circ} K=X$ if and only if $F(K)=Y, 3^{0}$ the function $F$ is complementative and additive [totally additive] ${ }^{1}$ ).

1) This theorem is due to A. Tarski. 
This theorem may be easily derived from the following remark: if a class $R$ of sets is a ring [a total ring], then the notions of $1^{0}$ empty set, $2^{0}$ whole space, $3^{0}$ sum of two sets [of an arbitrary finite or transfinite number of sets] belonging to $\mathbf{R}$ can be defined by using only the relation of inclusion between sets belonging to R. For instance, the sum of two sets $A_{1}$ and $A_{2}$ belonging to $\mathrm{R}$ is an element $B \in \mathbf{R}$ such that we have $R \supset B$ for $R \in \mathbf{R}$ if and only if $R \supset A_{1}$ and $R \supset A_{2}$.

Theorem (ii) justifies the following definition: A transformation $F(K)$ of a class $\mathrm{K}$ of sets into a class $\mathrm{L}$ is called isomorphism ${ }^{1}$ ) [total isomorphism ] if and only if there exists a weak isomorphism $G(K)$ between classes $\mathrm{K}_{0}$ and $\mathrm{L}_{0}$ [classes $\mathrm{K}_{t}$ and $\mathrm{L}_{t}$ ] such that $G(K)=F^{\prime}(K)$ for $K \in \mathrm{K}$.

I shall give one more definition: A transformation $F(K)$. of a class $\mathrm{K}$ of subsets of $X$ into a class $\mathrm{L}$ of subsets of $Y$ is called equivalence of $\mathrm{K}$ and $\mathrm{L}$ if there exists a biunivocal transformation. $\varphi(p)$ of $X$ into $Y$ such that $\varphi(K)=F(K)$ for each $K \in \mathbf{K}$.

We say that two classes $\mathrm{K}$ and $\mathrm{L}$ of sets are weally isomorphic [isomorphic, totally isomorphic, equivalent] when there exists a weak isomorphism [isomorphism, total isomorphism, equivalence] of $\mathrm{K}$ and $L$.

It is obvious that

(iii) Each equivalence is a total isomorphism, each total isomorphism is an isomorphism, each isomorphism is a weat isomorphism.

The isomorphisms and the equivalence may be defined also for systems of sets: two systems (with the same set $E$ of indexes) $\left\{A_{\xi}\right\}_{\xi \in \mathbb{Z}}$ and $\left\{B_{\xi}\right\}_{\xi \in \mathbb{Z}}$ are weakly isomorphic [isomorphic, totally isomorphic, equivalent $]^{2}$ ) if there exists a weak isomorphism [isomorphism, total isomorphism, equivalence] $F$ of the class of all sets $A_{\S}$ and the class of all sets $B_{\xi}$ such that $F^{\prime}\left(A_{\xi}\right)=B_{\xi}$ for each $\xi \in \Xi$.

\section{Obviously:}

(iv) Two classes of sets $\mathrm{K}$ and $\mathrm{L}$ are weakly isomorphic [isomorphic, totally isomorphic, equivalent] if and only if they can be well ordered in such a way that the obtained transfinite sequenoes of sets are weakly isomorphic [isomorphic, totally isomorphic, equivalent].

1) Cf. Kuratowski-Posament [1], p. 282.

2) The notion of equiralence of sequences of sets is duo to Jlam. (lf. Szpilrajn [2].

\subsection{Classes containing all one-element sets.}

(i) Let $\mathrm{K}$ and $\mathrm{L}$ be classes of subsets of non-void spaces $X$ and $Y$. If $\mathrm{K}$ and $\mathbf{L}$ contain all the one-element subsets of $X$ and $Y$ respectively, then each weak isomorphism between $\mathbf{K}$ and $\mathbf{L}$ is an equivalence between these classes.

The case $\overline{\bar{X}}=1$ being trivial, we may suppose $\overline{\bar{X}}>1$. Let $F$ be a weak isomorphism between $\mathbf{K}$ and $\mathbf{L}$. First let us prove that

$$
F[(p)] \neq 0 \text { for each } p \in X .
$$

The relation $F\left[\left(p^{\prime}\right)\right] \supset F[(p)]$ for $p, p^{\prime} \in X$ implies the relation $\left(p^{\prime}\right) \supset(p)$ and consequently $p^{\prime}=p$. Hence we have the relation $\left(^{*}\right)$ with the help of which we shall obtain the relation

$$
\overline{\overline{F[(p)}]}=1 \text { for each } p \in X .
$$

For this purpose suppose $(q) \subset F[(p)]$. Then, $F^{-1}$ being also a weak isomorphism, we have: $F^{-1}[(q)] \subset(p)$ and, :by $\left(^{*}\right), F^{-1}[(q)] \neq 0$. Hence $F^{-1}[(q)]=(p)$ or, otherwise, $F[(p)]=(q)$.

It follows from the equality $\left(\begin{array}{c}* \\ *\end{array}\right)$ applied for the functions $F$ and $F^{-1}$ that for each $x \in X$ there exists exactly one $y \in Y$ such that

$$
F[(x)]=y
$$

and vice versa: for each $y \in Y$ there exists exactly one $x \in X$ for which we have $\left({ }^{*}{ }^{*}\right)$. Now let us put $\varphi(x)=y$ whenever the equality $\left({ }^{*}{ }^{*}\right)$ holds; in that way we obtain a biunivocal transformation $\varphi$ of $X$ into $Y$. Consequently, it remains to prove that

$$
F(K)=\varphi(K) \quad \text { for each } \quad K \in \mathbf{K} .
$$

First we have

$$
\varphi(K)=\sum_{p \in K}[\varphi(p)]=\sum_{p \in K} F[(p)] C F(K),
$$

where the last relation follows directly from the fact that $F$ is a weak isomorphism.

On the other hand, suppose $q \in F(K)$. There exists an element $p \in X$ such that $q=\varphi(p)$ or, otherwise, $(q)=F[(p)]$. The function $F$ being a weak isomorphism, $(p) C K$; hence $q \in \varphi(K)$ and finally $\varphi(K) \supset F(K)$. Thus the relation $\left(\begin{array}{r}* * \\ *\end{array}\right)$ holds, and hence the theorem is proved. 
Theorem (i) has different consequences:

(ii) Two topological spaces are homeomorphic if and only if the classes of their closed subsets are wealily isomorphic.

(In other words: the type of partial order established by the relation of proper inclusion in the class of all closed subsets of a topological space determines its topology.)

(iii) There exists a generalized homeomorphism between $X$ and $Y$ [in the sense of Kuratowsti $\left.\left.i^{1}\right)\right]$ if and only if the classes of Borel sets in $X$ and in $Y$ are weakly isomorphic.

In order to prove Theorem (ii) [Theorem (iii)] it suffices to apply (i) and to remark that each equivalence between the classes of all closed [Borel] sets determines a homeomorphism [a generalized homeomorphism].

Applying the theorem on the non-equivalence of the class of sets measurable $(L)$ in the interval $I$ and of that of sets which possess the property of Baire (in $I)^{2}$ ); we obtain, in virtue of (i):

(iv) The olass of measurable sets in $I$ and that of sets possessing the property of Baire in I (in the large sense) are not weakly isomorphic.

\section{§ 2. Constituents and atoms.}

2.1. Definitions and fundamental properties. Let $\mathrm{K}=\left[K_{\xi}\right]_{\tilde{\xi} \in \Xi}$ be a system of sets. Each set of the form $K_{\xi_{1}}^{i_{1}} \cdot K_{\xi_{1}}^{i_{2}} \cdot \ldots \cdot K_{\xi_{n}}^{i_{n}}$, where $i_{1}, i_{2}, \ldots, i_{n}$ is a finite sequence of numbers 0 and $1^{3}$ ) and $\xi_{1}, \xi_{2}, \ldots, \xi_{n}$ is a finite sequence of distinct elements of $\Xi$, is called a constituent of $\mathrm{K}$. Each set of the form

$$
\prod_{\xi \in \Xi} K_{\xi}^{i \xi}
$$

where $\left\{i_{\xi_{5}}\right\}_{\Sigma_{5}}$ is a system consisting only of the numbers 0 and 1 , is termed an atom of $\mathbf{K}^{\mathbf{4}}$ ).

1) For the notion of generalized homeomorphism (homeomorphism of clase $(\alpha, \beta))$ see Kuratowski [1], p. 221 and [2].

2) Szpilrajn [1], p. 306

3) For the notation $Z^{0}$ and $Z^{1}$ see p. 134 .

4) For the notions of atom and constituent see e.g. Tarski [1], p. 236 and Kuratowski-Posament [1], p. 283 .
Let $L=\left\{L_{\xi \xi \xi \in \Xi}\right.$ be another system of sets (with the same set $\Xi$ of indexes). Constituents of $\mathbf{K}$ and $\mathbf{L}$ with the same indexes:

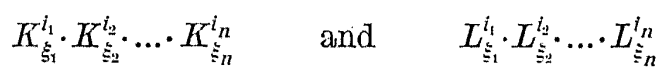

and respectively atoms

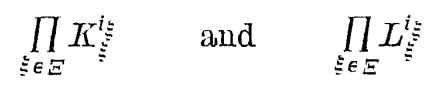

are called mutually corresponding.

The notions of atom and constituent of a transfinite sequence of sets (but not of corresponding atoms and constituents) do not depend on the order of elements in the considered sequence and consequently they can be also applied for classes of sets.

The following theorems are easily derived from the above given definitions:

(i) Each set belonging to $\mathbf{K}$ is a constituent of $\mathbf{K}$. Each two sets $K_{\xi}$ and $L_{\xi}$ (with the same index $\xi$ ) are corresponding constituents of $\mathbf{K}$ and $\mathbf{L}$.

(ii) The class consisting of the empty set and of all finite sums of constituents of $\mathbf{K}$ is equal to $\mathbf{K}_{\mathbf{0}}$.

(iii) Let $T_{1}$ and $U_{1}, T_{2}$ and $U_{2}$ be sums of a finite number of corresponding constituents of $\mathbf{K}$ and $\mathbf{L}$. Then $T_{1}-T_{2}$ and $U_{1}-U_{2}$ can be also represented as sums of corresponding constituents of $\mathbf{K}$ and $\mathbf{L}$.

(iv) Each two sets $K_{\xi}$ and $L_{\xi}$ can be represented as sums of a (finite or transfinite) number of corresponding atoms of $\mathbf{K}$ and $\mathbf{L}$.

(v) For each two different systems $\left\{i_{\xi}\right\}_{\xi \in \mathbb{Z}}$ consisting of the numbers 0 and 1 , the atoms $\left({ }^{*}\right)$ are disjoint; the union of all atoms is equal to the whole space.

(vi) The class consisting of the empty set and of all sums of atoms of $\mathbf{K}$ is equal to $K_{t}$. Each non-void element of the class $\mathbf{K}_{t}$ may be represented as a sum of non-void atoms of $\mathbf{K}$ in one way only. 
2.2. Constituents and atoms; isomorphisms and equivalence. Let us denote as above by $\mathrm{K}=\left\{K_{\xi}\right\rangle_{\xi \in g}$ and $\mathrm{L}=\left\{L_{\xi}\right\}_{\xi \in \Xi}$ two systems of sets (the indexes of which form the same set).

(i) Two systems of sets are isomorphic if and only if any two corresponding constituents are both void or both non-boid ${ }^{ }$).

Necessity. $K$ and $L$ being isomorphic, there existis and isomorphism $F$ between $\mathrm{K}_{0}$ and $\mathrm{L}_{0}$ such that $F\left(K_{\xi}\right)=L_{\xi}$. For each

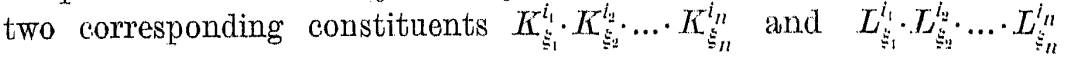
we have by 1.1 (ii, $3^{0}$ ):

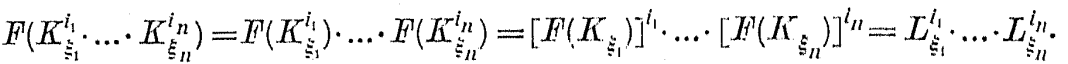

Thus, it follows from $1.1\left(\mathrm{ii}, 1^{0}\right)$ that

$$
K_{\xi_{s}}^{i_{1}} \cdot \ldots \cdot K_{\xi_{n}}^{i_{n}}=0 \text { if and only if } L_{s_{1}}^{i_{1}}, \ldots \cdot L_{\xi_{n}}^{l_{n}}=0 .
$$

Sufficiency. Suppose that any two corresponding constituents of $K$ and $\mathbf{L}$ are both void or both non-void.

We establish a relation $\varrho$ between each two sets $V_{1}+V_{2}+\ldots+V_{n}$ and $W_{1}+W_{2}+\ldots+W_{n}$, where $V_{j}$ and $W_{j}$ are two corresponding constituents of $K$ and $L$.

By 2.1 (ii), the domain of $\varrho$ is the ring $\mathbf{K}_{0}$, and the counterdomain of $\varrho$ is $L_{0}$. In particular, by virtue of 2.1 (ii), we have $K_{\xi} \varrho L_{\xi}$ for every $\xi$.

On account of 1.1 (i), in order to show $\mathbf{K}$ and $\mathbf{L}$ isomorphic, it remains to prove that for every two finite sequences $V_{1}, \ldots, V_{l i}$; $V_{1}^{\prime}, \ldots, V_{l}^{\prime}$ and $W_{1}, \ldots, W_{k} ; W_{1}^{\prime}, \ldots, W_{l}^{\prime}$ of corresponding constituents of $\mathrm{K}$ and $\mathbf{L}$, we have

$$
\sum_{j=1}^{k} V_{j} \subset \sum_{j=1}^{l} V_{j}^{\prime} \quad \text { if and only if } \sum_{j=1}^{k} W_{i} \subset \sum_{j=1}^{l} W_{j}^{\prime}
$$

or in other words:

$\left(^{*}\right) \quad \sum_{j=1}^{k} V_{j}-\sum_{j=1}^{l} V_{j}^{\prime}=0 \quad$ if and only if $\quad \sum_{j=1}^{k} W_{j}-\sum_{j=1}^{l} W_{j}^{\prime}=0$.

The proposition $(*)$ is an immediate consequence of the hypothesis and of 2.1 (iii).

(ii) Two systems of sets are totally isomorphic if and only if any two corresponding atoms are both void or both non-void.

$\left.{ }^{1}\right)$ Theorem due to Kuratowski-Posament [1], p. 283.
Necessity. $K$ and $\mathbf{L}$ being totally isomorphic systems of sets, there exists a total isomorphism $F$ between $\mathbf{K}_{t}$ and $\mathbf{L}_{t}$ such that $F\left(K_{\xi}\right)=L_{\S}$. For each two corresponding atoms of $\mathrm{K}$ and $\mathrm{L}$ :

$$
\prod_{\xi \in \Xi} K_{\xi}^{i} \quad \text { and } \quad \prod_{\tilde{\xi} \in \Xi} L_{\xi}^{i_{\xi}}
$$

we have by $1.1\left(\mathrm{ii}, 3^{0}\right)$ :

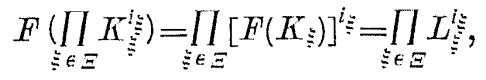

and by $1.1\left(\mathrm{ii}, 1^{0}\right)$ :

$$
\prod_{\xi \in \Xi} K_{\tilde{\xi}}^{i}=0 \text { if and only if } \prod_{\Xi \in \Xi} L_{\Sigma}^{i}=0 \text {. }
$$

Sufficiency. Let us suppose that every two corresponding atoms of $\mathrm{K}$ and $\mathbf{L}$ are both void or both non-void. We establish a relation $Q$ between each two sums $\sum_{i_{i}} A_{i_{i}}$ and $\sum_{i_{i}} B_{r_{i}}$ of corresponding non-void atoms of $K$ and $L$.

By 2.1 (vi) the domain and the counterdomain of the relation o are the total rings $\mathbf{K}_{t}$ and $\mathbf{L}_{t}$ respectively. In particular, by 2.1 (iv), we have $K_{\xi} \varrho L_{\Sigma}$ for each $\xi \in \Xi$.

It follows easily from $2.1(\mathrm{v})$ and (vi) that the relation $\varrho$ is a weak isomorphism between $\mathbf{K}_{t}$ and $\mathbf{L}_{t}$ and that, consequently, the classes $\mathbf{K}$ and $\mathbf{L}$ are totally isomorphic.

(iii) Two systems of sets are equivalent if and only if any two corresponding atoms are of the same power ${ }^{1}$ ).

Necessity. By hypothesis: $\mathrm{K}=\left\{K_{\xi}^{\prime}, \mathrm{L}=\left\{L_{\xi}\right\}, K_{\xi} \subset X, L_{\grave{c}} \subset Y\right.$, and $L_{\xi}=\varphi\left(K_{\xi}\right)$, where $\varphi$ is a biunivocal transformation of $X$ into $X$. It is obvious that $\varphi$ carries each atom of $\mathbf{K}$ into the corresponding atom of $\mathrm{L}$ and, consequently, these atoms are of the same power.

Sufficiency. It is a consequence of $2.2(\mathrm{v})$ and of the following remark easy to prove:

Let $X=\sum_{r_{i}} X_{i_{i}}$ and $Y=\sum_{r_{i}} Y_{i_{i}}$ be two decompositions of two spa. ces $X$ and $Y$ into disjoint sets such that $\overline{\bar{X}}_{i_{i}}=\overline{\bar{Y}}_{1_{i}}$. Then there exists a biunivocal transformation $q$ of $X$ into $Y$ such that $q\left(X_{i}\right)=Y_{i ;}$ for each $\%$.

$\left.{ }^{1}\right)$ It is a simple ganeralization of a theorem proved in the paper Szpilrajn [2], 2.5 (ii), p. 311 . 
An immediate corollary of Theorem (ii) and (iii) is:

(iv) If two totally isomorphic systems of sets $\mathbf{K}$ and $\mathbf{L}$ possess only atoms every of which contains at most a single point, then $\mathbf{K}$ and $\mathbf{L}$ are equivalent.

\section{§ 3. Enumerable sequences and classes of sets.}

3.1. Regular constituents. For each sequence $e=\left\{E_{n}\right\}$ every constituent of the form $E_{1}^{i_{1}} \cdot E_{2}^{i_{2}} \ldots \cdot E_{n}^{i_{n}}$ (where $i_{h}=0$ or 1 ) will be termed regular. Let us consider an arbitrary constituent $J=E_{k_{1}}^{j_{1}} \cdot E_{k_{q}}^{j_{2}} \cdot \ldots \cdot E_{k_{m}}^{j_{m}}$ of $e$; obviously, we may suppose $k_{1}<k_{2}<\ldots<k_{m l}$. Denote by 1 the class of regular constituents $H_{1}^{i_{1}} \cdot E_{2}^{l_{2}} \cdot \ldots \cdot E_{n}^{l_{n}}$ such that $n=k_{m}$ and $i_{k_{l}}=j_{l}$ for $l=1,2, \ldots, m$. All sots bolonging to $\mathrm{J}$ are disjoint and their sum is equal to $J$. Thus we obtain the following statements:

(i) Every constituent of a sequence $e$ of sets is the sum of a finite number of disjoint regular constituentis of $e$.

(ii) Each two corresponding constituents of two sequences $a$ and $b$ can be represented as the sums of a finite number of disjoint corresponding regular constituents of $a$ and $b$ respectively.

With the help of (ii) and 2.2 (i) we obtain:

(iii) Two sequences of sets are isomorphic if and only if any two corresponding regular constituents are both void or both non-void.

3.2. The characteristic function of isomorphic or equivalent sequences of sets. With the help of theorems on the characteristic function (CF 1.4 (v), 2.3 (iv), 2.3 (iii)) and the above proved theorems on isomorphisms and equivalence (3.1. (iii), 2.2 (ii) and 2.2 (iii)) we may state that

$A$ sequence $a=\left\{A_{n\}}\right\}$ of subsets of $X$ and a sequence $b=\left\{B_{n}\right\}$ of subsets of $\bar{Y}$ are (i) isomorphic, (ii) totally isomorphic, (iii) equivalent if and only if (i) $\overline{c_{a}(X)}=\overline{c_{b}(Y)}$, (ii) $c_{a}(X)=c_{b}(Y)$, (iii) $\overline{\overline{c_{a l}^{-1}(t)}}=\overline{c_{b}^{-1}(t)}$ for each $t \in C$.

3.3. Universal classes. An enumerable class $\mathbf{U}$ of sets is said to be universal in the sense of isomorphism [total isomorphism] when for each sequence $e$ of sets there exists a certain sequence of sets belonging to $\mathbf{U}$ which is isomorphic [totally isomorphic] to $e$. (i) The class $\mathbf{U}$ of sets both open and closed in $C^{1}$ ) is universal in the sense of isomorphism (Mostowski ${ }^{2}$ )-Kuratowski).

Let $e$ be a sequence of subsets of a space $X$ and let us put $T=\overline{c_{e}(\bar{X})}$. Since the set $T$ is closed in $C$, there exists a continuous function $f$ such that $\left.f(C)=T^{3}\right)$. By Theorem CF 2.4 there exists a sequence $v=\left\{\nabla_{n}\right\}$ of subsets of $O$ such that $e_{v}=f$. The function $c_{v}$ being continuous, all the sets $V_{n}$ are open-and-closed in $C$ (in virtue of CF $\left.3.7\left(\mathrm{iv}, 1^{0}\right)\right)$, i. $\theta$. they belong to $\mathrm{U}$. By 3.2(i) the relation $c_{v}(C)=\overline{c_{e}(X)}$ implies the existence of an isomorphism between the sequences $e$ and $v$.

C. Kuratowski proves this theorem directly, as follows:

Let $e=\left\{E_{n}\right\}$ be a given sequence of sets. For each interval $J$ of the Cantor set $O$ we denote by $J_{0}$ and $J_{1}$ its left and right half. Further we put $J_{0}=J_{1}=0$ for $J=0$. We shall define by complete induction a function which associates with each set of the form $E_{1}^{i_{1}} \cdot E_{2}^{i_{2}} \cdot \ldots \cdot E_{n}^{i_{n}}$ an interval of $O$ (or the empty set, or else the set $O$ ):

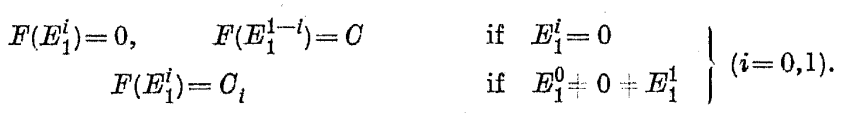

Denoting $F\left(E_{1}^{i_{1}} \cdot \ldots \cdot E_{n}^{i_{n}}\right)$ by $J$ we further put:

$$
\left.\begin{array}{c}
F\left(E_{1}^{i_{1}} \cdot \ldots \cdot E_{n}^{i_{n}} \cdot E_{n+1}^{i}\right)=0 \quad F\left(E_{1}^{i_{1}} \cdot \ldots \cdot E_{n}^{i_{n}} \cdot E_{n+1}^{1-i}\right)=J \text { if } E_{1}^{i_{1}} \cdot \ldots \cdot E_{n}^{i_{n}} \cdot E_{n+1}^{i}=0 \\
F\left(E_{1}^{i_{1}} \ldots \cdot E_{n}^{i_{n}} \cdot E_{n+1}^{i}\right)=J_{l} \text { if } E_{1}^{i_{1}} \cdot \ldots \cdot E_{n}^{i_{n}} \cdot E_{n+1}^{i} \div 0 \neq E_{1}^{i_{1}} \cdot \ldots \cdot E_{n}^{i_{n}} \cdot E_{n+1}^{1-i}
\end{array}\right\}(i=0,1)
$$

Finally we put

$$
G\left(E_{1}\right)=F\left(E_{1}\right), \quad G\left(E_{n}\right)=\sum_{\left(i_{1}, \ldots, i_{n-1}\right)} F\left(E_{1}^{i_{1}} \cdot \ldots \cdot E_{n-1}^{i_{n}-1} \cdot E_{n}\right) \quad(n=2,3, \ldots),
$$

where the system $\left(i_{1}, i_{2}, \ldots, i_{n-1}\right)$ runs through all sequences consisting of $n-1$ numbers equal to 0 or 1 .

Consequently, the function $G$ attaches to each set $E_{n}$ an open-and-closed subset of $C$. It follows easily from 3.2 (iii) that the sequences $\left\{E_{n}\right\}$ and $\left\{G\left(E_{n}\right)\right\}$ are isomorphic.

(ii) In any space of the power $c$ there exists $2^{c}$ sequences no two of which are totally isomorphic.

1) This class is enumerable; see p. 134

2) Mostowski [1], Korollar 5, p. 46 .

s) because every compact space is a continuous image of $C$; cf. e.g. Haus. doxff $[1]$, p. 197. 
Let be $\overline{\bar{X}}=c$. For each non-void set $T C O$ there exists a function $f$ such that $f(X)=T$ and, consequently (by CF 2.4), a sequence $e$ of sets $E_{n} \subset X$ such that $c_{e}(X)=T$. Since the power of the class of subsets of $C$ is equal to $2^{c}$, we obtain our theorem by applying Theorem 3.2 (ii).

(iii) There exists no enumerable class universal in the sense of total isomorphism ${ }^{1}$ ).

It is a consequence of (ii) and of the fact that for each enumerable class $E$ of sets there exists only $c$ sequences of sets belonging to $E$.

\subsection{Measurability $(B)$.}

(i) For each sequence $e=\left\{E_{n}\right\}$ of subsets of $X$ there exists a set TCC such that $e$ is totally isomorphic to a sequence of sets both open and closed in $T$. More precisely: The operation $0_{a}^{-1}$ is a total isomorphism between the sequences $\left\{c_{2}(X) \cdot C_{n\}}\right\}$ and $\left\{E_{n}{ }^{p}\right.$.

It is an immediate consequence of the properties of the characteristic function and those of the operation of the counterimage (CF 2.5).

(ii) A sequence $e=\left\{E_{n\}}\right\}$ of subsets of $X$ is totally isomorphic to a sequence of Borel subsets of a Borel space (or: of sets which are both $G_{\delta}$ and $F_{\sigma}$ in $I$, or: of sets which are both open and closed, in $N$ ) if and only if the set $c_{e}(X)$ is analytic.

Necessity. Let $e=\left\{E_{n}\right\}$ be a sequence of subsets of $X$ which is totally isomorphic to a sequence $b=\left\{B_{n}\right\}$ of Borel subsets of a Borel space $Y$. Thus the function $c_{b}$ is measurable $(B)$ (CF $3.7\left(\mathrm{iv}, 3^{\circ}\right)$ ) and consequently the set $c_{b}(Y)$ is analytic. By Theorem 3.2. (ii), the set $c_{2}(X)$ is analytic as well.

Sufficiency. Suppose that $e=\left\{E_{n}\right\}, E_{n} \subset X$ and that the set $c_{i}(X)$ is analytic. Then there exists 1$)$ a function $f$ of the first class such that $f(I)=c_{e}(X)$ and 2) a continuous function $g$ such that $g(N)=c_{e}(X)$. By CF 2.4 there exists 1) a sequence $a=\left\{A_{n}\right\}$ of subsets of $I$ such that $c_{a}=f$ and 2) a sequence. $b=\left\{B_{n}\right\}$ of subsets of $N$ such that $c_{b}=g$. By CF 3.7 (iv, $1^{0}, 2^{0}$ ) 1) all the sets $A_{n}$ are both $F_{\sigma}$ and $G_{\delta}$ in $I$ and 2) all the sets $B_{n}$ are both open and closed in $N$. Finally, by Theorem 3.2 (ii), the sequences $a$ and $b$ are totally isomorphic to $e$. p. 313

1) and a fortiori in the sense of equivalence; of. szpilraju [2], 2.6(v),
The problem of characterizing in a similar way the sequences of sets which are equivalent to sequences of Borel sets is still open. We shall give only certain partial answers:

(iii) If a sequence $e=\left\{E_{n}\right\}$ is equivalent to a sequence of Borel subsets of $I$, then the set

$$
\left.Z_{e}(\mathfrak{n})=\underset{t}{E\left[\overline{e_{e}^{-1}(t)}\right.}=\mathfrak{n}\right]
$$

is analytic for $\mathrm{n}=\mathrm{c}$ and its complement $C-Z_{e}(\mathfrak{n})$ is analytic for $\mathfrak{n}=0,1,2, \ldots, \mathrm{s}_{0}$.

It is a simple consequence of the statements 3.2 (iii), CF 3.7 (iv, $\left.3^{0}\right)$ and of the following theorem: For each function $f$ measurable $(B)$ the set $E\left[f^{-1}(t)=n\right]$ is analytic for $n=c$ and its complement is analytic for ${ }^{t}=0,1,2, \ldots, s_{0}$.

This last theorem for $n=0$ follows immediately from the analyticity of the set $f(I)$ and from the equality

$$
f(I)=C E\left[\overline{\overline{f^{-1}(t)}}=0\right] .
$$

The same theorem with $\mathfrak{n}=1$ was stated by Lusin ${ }^{1}$ ) and the cases with $\mathfrak{n}=2,3, \ldots$ may be easily deduced from his result.

The case of $\mathfrak{n}=\boldsymbol{\aleph}_{0}$ is due to Miss Braun ${ }^{2}$ ) and that of $\mathfrak{n}=\mathrm{c}$ to Mazurkiewiez and Sierpiński ${ }^{3}$.

We do not know whether the condition of Theorem (iii) is sufficient. This problem is reducible to the question of inversion of the theorem just quoted: riven a decomposition into disjoint sets: $I=A+B+E_{0}+E_{1}+\ldots$; when does there exist a real function $f$ measurable $(B)$ such that:

$$
\begin{aligned}
& \left.E_{n}=E \overline{\left[f^{-1}(y)\right.}=n\right] \\
& \left.\left.A=\underset{y}{E\left[\overline{f^{-1}(y)}\right.}=\mathrm{c}\right], \quad B=\underset{y}{E\left[\overline{f^{-1}(y)}\right.}=\aleph_{0}\right] \quad \text { ? }
\end{aligned}
$$

In particular: is the necessary condition of analyticity of the sets: $A, I-B$ and $I-E_{n}(n=0,1,2, \ldots)$ also sufficient?

(iv) Let $e=\left\{E_{n}\right\}$ be a sequence of subsets of a space $X$. Let us suppose that $\overline{\bar{X}}=c$ and that each atom of $e$ contains at most a single point. Then the sequence $e$ is equivalent to a sequence of Borel subsets of $I$ [or: of open-and-closed subsets of $\left.N^{*}\right]$ if and only if the set $c_{e}(X)$ is a Borel set.

1) Gf. e.g. Lusin [1], p. 259 and Kuratowski [1], p. 259.

2) Braun [1], Théorème 7, p. 171.

s) Cf. e. g. Kuratowski [1], p. 262. 
In order to prove this theorem it suffices to apply Theorems CF 3.6, CF 3.7 (iv), and the following: a linear set $E$ of the power $c$ is a Borel set if and only if there exists a biunivocal function $f$ measurable $(B)$ such that $f(I)=E^{1}$ ) (or: a continuous biunivocial function $g$ such that $\left.g\left(N^{*}\right)=E^{2}\right)$ ).

( $v)$ There exists a sequence e of sets contained in $I$ which is totally isomorphic to a sequence of Borel subsets of $I$, but equivalent to no such a sequence.

In order to build such a sequence it suffices to take a function $f$ such that $f(I)=C$ and for which the set $E\left[t \bar{f}^{-1}(\bar{t})=c\right]$ is not analytic. In virtue of $\mathrm{CF} 2.4$, there exists a sequence $e$ of subsets. of $I$ such that $c_{e}=f$. By Theorem (ii), it is totally isomorphic to a sequence of Borel subsets of $I$, and, by (iii), it is equivalent to no. such a sequence.

3.5. Projectivity. It is possible to consider problems analogous to those considered above concerning sequences of projective setis. Denote by $\mathbf{P}_{0}$ the class of analytic sets, by $\mathbf{C}_{n}$ the class of complements of all sets belonging to $\mathbf{P}_{n}$, by $\mathbf{P}_{n+1}$ the class of all continuous images of sets belonging to $\mathbf{C}_{n}$, and finally put $\mathbf{B}_{n}=\mathbf{C}_{n} \cdot \mathbf{P}_{n}$ $(n=0,1,2, \ldots)$. We shall formulate a few theorems easy to prove:

(i) A sequence $e$ of subsets of a space $X$ is totally isomorphic to a sequence of subsets of $I$ belonging to the class $\mathbf{B}_{n}$ if and only if the set $c_{e}(X)$ belongs to the class $\mathbf{P}_{n}$.

(It is a generalization of Theorem 3.4(ii)).

(ii) A sequence $e$ of subsets of a space $X$ is totally isomorphic to a sequence of projective subsets of $I$ if and only if the set $c_{\rho}(X)$ is projective.

(iii) There exists a sequence of subsets of $I$ belonging to $\mathrm{B}_{n+1}$ which is totally isomorphic to no sequence belonging to $\mathrm{B}_{n}(n=0,1,2, \ldots)$.

(iv) There exists a sequence of sets which is totally isomorphic to a sequence of Borel subsets of $I$ and which is equivalent to no sequence of projective sets.

(v) There exists a sequence of sets which is totally isomorphic to no sequence of projective sets.

1) Cf. e. g. Kuratowski [1], p. 231.

${ }^{2}$ Cf. e.g. Szpilrajn [2], p. 313.
3.6. Convergence. Now we shall consider problems analogous to the preceding but only for sequences converging in the sense of the General Theory of Sets. We know that a sequence $e$ of sets converges if and only if the function $c_{e}$ assumes only values of the form $n / 3^{i n}$ (CF 3.4).

(i) Each convergent sequence of sets is totally isomorphic to a sequence of sets both $F_{\sigma}$ and $G_{\delta}$ in $I$ and to a sequence of sets both open and closed in $N$.

It is a simple consequence of the theorem just quoted and Theorem 3.4 (ii).

Moreover, let us remark that every sequence totally isomorphic to a convergent sequence is itself convergent.

(ii) In order that every convergent sequence of sets contained in $I$ be equivalent to a sequence of Borel sets [or: of sets which are both $F_{\sigma}$ and $G_{\partial} J$, it is necessary and sufficient that the hypothesis of the continuum be true ${ }^{1}$ ).

Necessity. Let $E$ be any subset of $I$. The sequence $E, E, \ldots$ is convergent and therefore it is equivalent to a sequence $B, B, \ldots$, where $B$ is a Borel subset of $I$. Hence $\bar{E}=\bar{B}$ and consequently $\bar{E} \leqslant \kappa_{0}$ or $\bar{E}=c$.

Sufficiency. It is a consequence of the above cited theorem, Theorem 3.2 (iii), and the following remark:

Let $f$ be a real function carrying $I$ into an at most enumerable set. If $\overline{f^{-1}(t)}=c$ or $\overline{f^{-1}(t)} \leqslant s_{0}$ for each real $t$; then there exists a real function $g$ of the first class defined on $I$ and such that $\overline{f^{-1}(t)}=\overline{\overline{g^{-1}(t)}}$ for each real number $t$.

To prove this, denote by $\left\{t_{n}\right\}$ the (finite or infinite) sequence of all values of the function $f$ and by $F_{n}$ a closed set contained in the open interval $(1 / n+1,1 / n)$ such that $\overline{\overline{F_{n}}}=\overline{\overline{f^{-1}\left(t_{n}\right)}}$. Obviously there exists a positive integer $n_{0}$ such that $\overline{\overline{f^{-1}\left(t_{n_{0}}\right)}}=c$. Putting $g(x)=t_{n}$ for $x \in F_{n}$ and $g(x)=t_{n_{0}}$ for $x \in I-\left(F_{1}+F_{2}+\ldots\right)$ we obtain the required function $g$.

1) (ff. the preliminary report Szpilrajn [5]. 


\section{References.}

Braun S. [1] Quelques théorèmes sur les cribles boreliens: Fund. Math. 20 (1933), p). 166-172.

Hausdorff $\mathrm{F}$. [1] Mengenlehre. Berlin-Leipzig 1935.

Kuratowski C. [1] Topologie I. Monografie Matematyczne 3, Warszawa. Lwów 1933.

- [2] Sur une généralisation de la notion d'homéomorphie. Fund. Math. 22 (1934), pp. 206-220.

Kuratowski C. et Posament T. [1] Sur l'isomorphie algébro-logique et les ensembles relativement boreliens. Fund. Math. 22 (1934), pp. 281286.

Lusin N. [1] Leçons sur les ensembles analytiques et leurs applioations. Collection Borel, Paris 1930.

Hostowski A. [1] Abzühlbare Boolesche Körper und ihre Lmondung an die allgemeine Metamathematil. Fund. Math. 29 (1937), pp. 3453.

Sierpiński W. [1] Hypothèse du continu. Monografie Natematyczno 4, Warszawa-Lwów 1934.

Stone M. H. [1] The theory of representations for Boolean alyebras. Truns. Amer. Math. Soc. 40 (1936), pp. 37-111.

Szpilrajn E. [1] Remarques sur les fonctions completement additives d'en semble et sur les ensembles jouissant de la propriété de Baire. Fund. Math. 22 (1934), pp. 303-311.

- [2] Sur l'équivalence des suiles d'ensembles et l'équivalence des fonstions. Fund. Math. 26 (1936), pp. 302-326. Correction... Fund. Math. 27 (1936), p. 294

- [3] On the equivalence of some classes of sets. Fund. Math. 30 (1938), pp. 235-241.

- [4] The characteristic function of a sequence of sets and some of its applications. Fund. Math. 31 (1938), pp. 207-22311).

- [5] Concerning convergent sequences of sets. Aml. Soc. Pol. Math. 17 (1938), p. 115 .

- [6] On the isomorphism and the equivalence of classes and sequences of sets. Ann. Soc. Pol. Math. 17 (1938), pp. 119-120.

Tarski A. [1] Sur les classes d'ensembles oloses par rapport à certaines opérations élémentaires. Fund. Math. 16 (1930), pp. 181-304.

- [2] Über additive und multiplikative Mengenkörper und Mengenfunktionen. Comptes-rendus Soc. Sc. Varsovie 30 (1937), pp. 151-181.

Whitehead A. N. and Russell B. [1] Principia Mathematica, vol. II. Cambridge 1927.

1) This paper is refered to as CF.

\section{On the asphericity of regions in a 3-sphere.}

By

\section{J. H. C. Whitehead (Oxford).}

1. This note arises out of an attempt to answer two questions proposed by $\mathrm{S}$. Eilenberg ${ }^{1}$ ), namely:

1. pour quelles courbes simples fermées $\Omega_{0} C S^{3}$ l'ensemble $S^{3}-\Omega_{0}$ est asphérique?

2. pour quels couples $\Omega_{1}, \Omega_{2} \subset S^{3}$ de courbes simples fermées disjointes l'ensemble $S^{3}-\left(\Omega_{1}+\Omega_{2}\right)$ est asphérique?

I first show how Reidemeister's theory") of "Homotopiekettenringe" can be applied to the study of the first question in case $\Omega_{0}$ is a polygonal knot, which I will call $k$, and in $4 \mathrm{I}$ show how the methods of $\mathbf{2}$ and $\mathbf{3}$ can be applied to the study of similar questions. Some examples are given in $\mathbf{5}$, and $\mathbf{6}$ contains an "addition theorem" with an application to the study of knots and linkages. Taking $M^{3}=S^{3}$ it follows from theorem 6 , in 7 , that the hypothesis " $S^{3}-k$ is aspherical", $k$ being any polygonal knot in a 3 -sphere $S^{3}$, implies the algebraic analogue of Dehn's lemma ${ }^{3}$ ) for circuits in $S^{3}$ (i.e. it implies that, if $k$ bounds a singular 3-cell without singularities on the boundary, then $\pi_{1}\left(S^{3}-k\right)$ is cyclic.) The final section is an appendix on the group ring of an "indexed" group ${ }^{4}$ ).

1) Fund. Math., 28 (1937), p. 241. We recall that a space $X$, is called aspherical (W. Hurewicz, Proc. Akad. Amsterdam, 39 (1936), p. 215) if all the (additive) higher homotopy groups $\pi_{n}(X), n>1$, reduce to zero. $\pi_{1}(X)$ is the (multiplioutive) fundamental group, which need not reduce to $I$.

2) See: Abh. Math. Sem. Hamburg, 10 (1934), p. 211; Journal für die r.u.a. Math., 173 (1935), p. 164, and other papers.

3) Math. Annalen, $69(1910)$, p. 147. There is a gap in Delm's argument (at the top of p. 151) which has not yet been filled. See also E. Pannwitz, Math. Amalen, 108 (1933), p. 629 (\$3), and I. Johansson, Math. Annalen, 110 (1934), p. 312 and 115 (1938), p. 658

4) J. W. Alexander, Trans. American Math. Soc., 30 (1928), 290. 